\title{
Release from proactive interference in rat spatial working memory
}

\author{
William A. Roberts ${ }^{1} \cdot$ Hayden MacDonald ${ }^{1} \cdot$ Lyn Brown $^{1} \cdot$ Krista Macpherson $^{1}$
}

Published online: 31 March 2017

(C) Psychonomic Society, Inc. 2017

\begin{abstract}
A three-phase procedure was used to produce proactive interference (PI) in one trial on an eight-arm radial maze. Rats were forced to enter four arms for reward on an initial interference phase, to then enter the four remaining arms on a target phase, and to then choose among all eight arms on a retention test, with only the arms not visited in the target phase containing reward. Control trials involved only the target phase and the retention test. Lower accuracy was found on PI trials than on control trials, but performance on PI trials significantly exceeded chance, showing some retention of target memories. Changes in temporal and reward variables between the interference, target, and retention test phases showed release from PI, but changes in context and pattern of arm entry did not. It is suggested that the release from PI paradigm can be used to understand spatial memory encoding in rats and other species.
\end{abstract}

Keywords Release from proactive interference $\cdot$ Spatial working memory $\cdot$ Radial maze $\cdot$ Rats

\section{Introduction}

Proactive interference (PI) has long been recognized as a major source of forgetting in human memory, both long-term memory (Postman, 1962) and short-term memory (Keppel \& Underwood, 1962). It arises when memories formed prior to the formation of a target memory then interfere with

William A. Roberts

roberts@uwo.ca

1 Psychology Department, Western University, London, Ontario, Canada N6A 5C2 subsequent retrieval of the target memory. A particularly interesting set of experiments carried out by Wickens and his colleagues (Goggin \& Wickens, 1971; Wickens, 1970; Wickens, Clark, Hill, \& Wittlinger, 1968) showed that a shift in the semantic category of words tested for short-term memory led to a substantial release from PI. Thus, subjects given four successive trials in which they had to remember sets of three words for $20 \mathrm{~s}$ while counting backwards from an arbitrary number showed substantial loss of memory over the four trials when the words came from the same semantic category, say fruits. If the semantic category was switched on the fourth trial, say to animals, there was a significant rise in subjects' ability to remember these words after $20 \mathrm{~s}$. It was suggested that words from the same semantic category may become easily confused in short-term memory, whereas memories of words from a different category are more easily discriminated and retrieved. Subsequently, release from PI in short-term memory was shown with changes along other dimensions, such as display size (Turvey \& Egan, 1969), the occupations of famous people (Darling \& Valentine, 2005), the language of word presentation (Dillon et al., 1973), and a change in presentation sensory modality (Kroll, Bee, \& Gurski, 1973).

Clear evidence of PI has also been found in memory experiments with non-human animals. Studies of long-term retention have revealed PI in rats (Gleitman \& Jung, 1963) and pigeons (Burr \& Thomas, 1972; Kraemer, 1984). Typically, these experiments initially trained animals to respond to one of two spatial or visual stimuli for reward, followed by reversal training in which choice of the alternative stimulus was rewarded. A control group learned only a single discrimination response. When tested for retention of the reversed discrimination, animals showed marked forgetting in relation to the control group, particularly at longer retention intervals. Theoretical accounts suggest that memory for the correct stimulus on the reversed discrimination became confused with 
memory for the stimulus correct on the original discrimination, thus leading to frequent incorrect choice on the retention test.

Similar experiments have been carried out to study PI in animal working memory. Working memory experiments test for retention of single events over relatively short retention intervals. Working memory in pigeons has often been tested using a delayed matching-to-sample procedure in which a sample stimulus (red or green) is shown on a center key and then extinguished. After a retention interval of a few seconds, red and green appear on side keys as comparison stimuli, and a pigeon must choose the key that matches its memory of the sample in order to receive food reinforcement. Working memory is often impaired when the incorrect comparison stimulus on Trial $n$ was the correct match to sample on Trial $n-1$. Thus, if red was the correct sample match on Trial $n-1$ and green is the correct sample match on the Trial $n$, PI arises through confusion between the memories for Trial $n$ and Trial $n-1$ (Grant, 1975). When only two stimuli are used as sample and incorrect comparison stimuli over repeated trials within a session, PI arises as memories from preceding trials interfere with matching the sample stimulus presented on the current trial. This intertrial PI is pronounced when a short intertrial interval separates trials and is alleviated by using a long intertrial interval (Roberts, 1980; Roberts \& Kraemer, 1982) that allows some forgetting of the interfering memories.

Proactive interference also arises in experiments that require same-different judgments between sample and comparison stimuli (Devkar \& Wright, 2016; Wright, Katz, \& Ma, 2012). Pigeons and monkeys have been presented with sample pictures that change from trial to trial. On each trial, a sample picture is followed by a retention interval and a comparison picture that is either the same as the sample or different from the sample. Pressing the comparison picture when it matches the sample or pressing an alternative white rectangle when a different comparison is shown yield reinforcement. On some test trials involving the presentation of a non-matching picture different from the sample, the test picture is one from the samples presented on preceding trials. Errors shown as same responses to different test pictures were most pronounced when the different picture came from the just preceding trial and decreased as the distance of the different picture in the preceding trials increased. In other words, subjects confused memory of the picture seen on a previous trial with memory of the picture seen on the current trial. Interestingly, this PI effect becomes more pronounced with longer delay intervals between the sample and test stimulus in experiments with pigeons (Wright et al., 2012) but not with monkeys (Devkar \& Wright, 2016). Release from PI has also been found in a rhesus monkey when the category of pictures tested on successive working memory tests was switched from flowers to faces of other non-human primates (Jitsumori, Wright, \& Shyan, 1989).
Working memory experiments carried out with rats also show PI effects. Rats are initially trained on trials that involve an initial forced left or right positional response, either to one side of a T-maze (Grant, 1980, 1981) or on one lever in an operant chamber (Dunnett \& Martel, 1990). A free-choice memory test is then given in which the rat can choose between the left or right response, with only choice of the side opposite the forced choice reinforced. Proactive interference is then examined by testing rats on two trials in a row, with rats forced to make a choice on Trial $n-1$ that is opposite the correct choice on Trial $n$. Thus, a rat might be forced to make a left response for reward on Trial n-1 followed by Trial $n$ on which it would need to make a right response to obtain reward. This procedure typically yields PI, shown as substantially lower accuracy on Trial $n$ of two-trial sequences than on single trial control tests that do not involve a preceding interference trial. It is often found in such experiments that PI is stronger at longer retention intervals (Dunnett \& Martell, 1990; Grant, 1981) and is reduced by increasing the intertrial interval (Grant, 1981).

Evidence of PI in rats has also been found for a more complex spatial memory task, navigating the radial-arm maze (Roberts \& Dale, 1981). The typical radial maze contains eight arms that radiate out from a central hub at equal angles and form a circular pattern. When rats are allowed to search each arm for food reward placed in a cup at the end of the arms, they enter all of the arms with little repetition of arms previously visited. A number of studies suggest that rats perform this task by remembering the places they have and have not visited. Although it was initially suggested that rats given repeated trials on the radial maze erased memory of each trial before the next trial (Olton, 1977, 1978), Roberts and Dale (1981, Experiment 1) showed that correct choices declined over repeated trials. This finding suggested that the carryover of interfering memories from previous trials caused PI with retention of arm entries on subsequent trials. Further, the loss of accuracy on repeated trials was most marked at later choices within a trial when the retention interval was long and the memory load was high. In a subsequent experiment, Roberts and Dale (1981, Experiment 2) forced rats to enter four randomly chosen arms for reward in an initial study phase of a trial and then allowed rats to choose freely among all eight arms in a test phase, with only the arms not previously entered baited with reward. When rats were given repeated study-test trials, accuracy of correct choice in the test phase dropped over trials when the delay between study and test was $60 \mathrm{~s}$ but not when the delay was 0 s. Cohen, Reid, and Chew (1994) reported similar effects; choice accuracy on a radial maze dropped substantially from Trial 1 to Trials 2 and 3 when trials were massed (2-min intertrial intervals) but not when trials were spaced (2-h intertrial intervals). All of these findings were in keeping with previously observed PI effects in human and animal experiments. Retention dropped across 
trials as interfering memories from previous trials accumulated, and memory loss was most marked at longer retention intervals.

In the experiments reported here, we used a procedure first reported by Hoffman and Maki (1986) that produced PI more immediately within a single trial. Rats were given an initial interference study phase on the radial maze that consisted of forced visits to four arms. They were then given a second target study phase that consisted of forced visits to the four arms not visited on the initial study phase. After a retention interval following the target study phase, a rat was allowed to choose freely among the eight arms, with reward placed only on the arms not visited in the target phase. Performance on this test phase was compared with that on control trials when only a single target study phase was given to find out if the memories encoded on the initial interference phase proactively interfered with retention of memories encoded on the target phase. An initial experiment used this paradigm to examine the effects on PI of time intervals between the interference and target study phases and of the length of the retention interval between the target phase and the test phase. In subsequent experiments, several variables were changed between the interference and target phases to see whether release from PI could be produced in rat spatial memory.

\section{Experiment 1}

In some earlier experiments carried out with monkeys, it was suggested that failure of temporal discrimination is an important factor in the production of PI (D'Amato, 1973; Worsham, 1975). That is, PI may arise from a failure to remember which occurred most recently, an interfering initial event or a subsequent target event. In general, the more these events are spaced apart temporally relative to the time of the retention test, the less PI should be found. The frequent observation that spacing trials apart reduces PI in working memory experiments is in keeping with the importance of temporal discrimination. As a rough indicator of the ease of temporal discrimination, D'Amato (1973) suggested calculating the ratio of the time interval between encoding an initial interfering memory and the retention test and the time interval between encoding the target memory and the retention test. As this ratio increases, PI should decrease.

To find out if rats would show PI within trials on the radial maze, three-phase trials were conducted in which a rat received an initial phase in which it was forced to enter four randomly chosen arms for food reward. These four arm entries constituted the encoding of a potentially interfering spatial memory. In the next phase of the trial, the rat encoded the target memory by forced entries into the remaining four arms of the maze for food reward. In the retention test phase of a trial, the rat was allowed to choose among all eight arms, with only the arms not entered during the target memory formation phase containing reward. Performance accuracy on the retention test was compared to performance on control trials that involved only the target memory formation and retention test phases. Significantly lower accuracy on three-phase trials than on two-phase control trials indicated PI.

To examine the temporal discrimination hypothesis with this paradigm, the intervals between the three phases were varied relative to one another. Two retention intervals were used, $5 \mathrm{~min}$ and $60 \mathrm{~min}$. Accuracy on control trials was measured at each of these retention intervals. At each of these retention intervals, two three-phase trials were examined in which the interval between the initial interference phase and the subsequent target phase was varied between $5 \mathrm{~min}$ and $60 \mathrm{~min}$. Thus, the four kinds of PI trials coded in terms of the successive intervals between interference phase and target phase and between target phase and retention test are 5-5, 60 $5,60-60$, and 5-60. The ratio between interference phase to retention test interval over target phase to retention test interval for these four conditions is $10 / 5=2,65 / 5=13,120 / 60=2$, and $65 / 60=1.08$, respectively. Clearly, the temporal discrimination ratio predicts the least PI in the 60-5 condition.

\section{Method}

\section{Subjects}

Twelve male Long-Evans rats that were approximately 100 days old at the beginning of the study were used. The rats were kept at $85 \%$ of their free-feeding weight (approximately $350 \mathrm{~g}$ ) prior to testing. Rats were pair-housed in standard polypropylene cages with water ad libitum on a 12:12 h light-dark cycle, with light onset at 7 am and offset at $7 \mathrm{pm}$. Rats were fed Pro Lab Rat Chow daily after testing in concordance with their target weights.

\section{Apparatus}

The apparatus used was a radial maze made of $2.5-\mathrm{cm}$ plywood. It contained a central octagonal hub with a diameter of $30.5 \mathrm{~cm}$ and eight arms that extended from the central platform, each $91.5 \mathrm{~cm}$ long and $7.5 \mathrm{~cm}$ wide, with equal angular distance between adjacent arms (see Fig. 1). Wooden legs elevated the maze $61 \mathrm{~cm}$ above the floor. White plastic food cups that were $3.5 \mathrm{~cm}$ in diameter and $1.5 \mathrm{~cm}$ deep were attached to the end of each arm. An additional food cup was placed in the middle of the central hub of the maze. The entire maze was painted light gray. White pieces of board $23 \mathrm{~cm} \times$ $21.5 \mathrm{~cm}$ were attached to the sides of the arms adjacent to the central hub of the maze to prevent rats from crossing between the arms. Gray wooden blocks measuring $7.5 \mathrm{~cm} \times 15 \mathrm{~cm}$ could be bolted to the arm entrances to prevent a rat from entering an arm during the study phase of a trial. Noyes 
PI Trial

Interference Phase

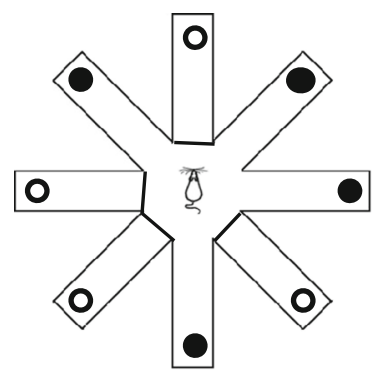

Target Study Phase

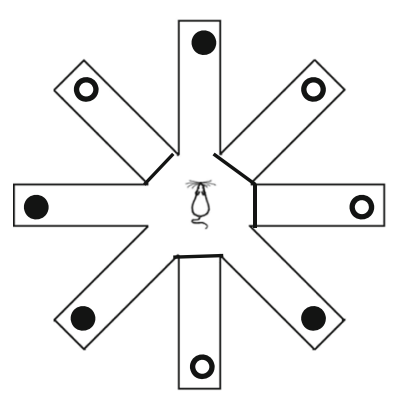

Retention Test Phase

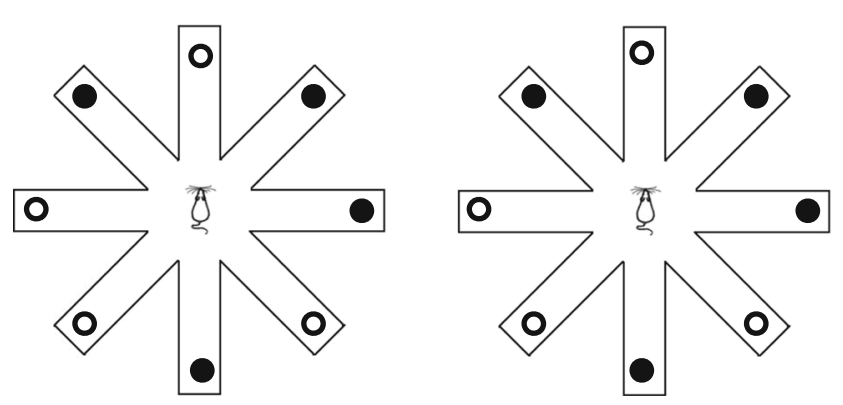

Fig. 1 Diagram of the maze setup on successive phases of PI and control trials. Lines across arm entrances indicate blocked arms

Precision sucrose Pellets (45 mg; PJAI-0045, Research Diets Inc., New Brunswick, NJ, USA) were used as food reward on the maze, with two pellets placed in the food cup on a rewarded arm. A single $60-\mathrm{W}$ bulb was used in a desk lamp for dim lighting in the corner of the $3.8 \mathrm{~m} \times 3.8 \mathrm{~m}$ testing room. The room contained a stool, two transport carts, pieces of apparatus piled in one corner, and posters on the walls. A white noise generator emitted white noise at $60 \mathrm{~dB}$ to mask extraneous sounds.

\section{Procedure}

After an initial period of placing the rats on the maze so that they became accustomed to eating the reward pellets from the food cups, training began. Rats were allowed to explore the maze with all arms baited on one trial for each of five days.
Experimental testing then began. In this experiment and all of the following experiments, rats were tested with one trial per day. On control trials, each rat was forced to enter four rewarded arms on an initial study phase. The four arms chosen differed among the 12 rats and were chosen by a random number generator. A rat was placed in the center of the maze with blocks placed in front of the openings to four arms, and reward placed in the four open arms. On all trials in this and subsequent experiments, the relevant reward was also placed in the central hub cup to act as a reminder of the rewards to be found on the correct arms. Rats always consumed this food in the center before entering an arm. After visiting all four arms, the rat was removed from the maze and kept in its cage for a retention interval. The retention interval was $5 \mathrm{~min}$ or $60 \mathrm{~min}$ on different sessions. For the retention test, the rat was replaced on the center of the maze with all eight arms open and with reward placed on the arms not entered during the study phase. The rat was allowed to enter arms until it had visited all of the arms containing reward. The experimenter recorded the sequence of arm entrances.

A similar procedure was followed on PI trials, but an initial potentially interfering study phase preceded the target study phase (see Fig. 1). A rat was forced to enter four randomly chosen rewarded arms and was returned to its cage. After an inter-phase interval of 5 or $60 \mathrm{~min}$, the rat was returned to the maze and forced to enter the remaining four arms for reward in the target study phase. It was then returned to its cage for the retention interval of 5 or $60 \mathrm{~min}$ and brought back to the maze for the retention test with all arms open but only the arms not visited in the target study phase rewarded. Thus, there were four combinations of inter-phase interval and retention interval that formed the PI trials, PI 5-5, PI 5-60, PI 60-60, and PI 60-5. Performance on these PI trials was compared with that on the appropriate control trials, Control 5 and Control 60.

The rats were tested for 6 days in the order Control 5, Control 60, PI 5-5, PI 60-5, PI 60-60, and PI 5-60. They were then tested for another 6 days in the reverse order of that used in the first 6 days.

\section{Results}

The dependent variable reported as a measure of memory accuracy on the retention test is the percentage of correct (rewarded) arms entered in the first four arms visited. Performance in the six testing conditions is shown in Fig. 2. These data suggest that performance was best in the control conditions, with lower accuracy in the PI conditions. Among the PI conditions, lowest accuracy is seen in PI 5-5, PI 60-60, and PI 5-60, with higher accuracy in PI 60-5.

A one-way within subjects analysis of variance (ANOVA) showed that there was a significant effect of condition, $F(5$, $55)=9.28, p<.01, \eta_{\rho}^{2}=.46$. Post-hoc Tukey HSD 


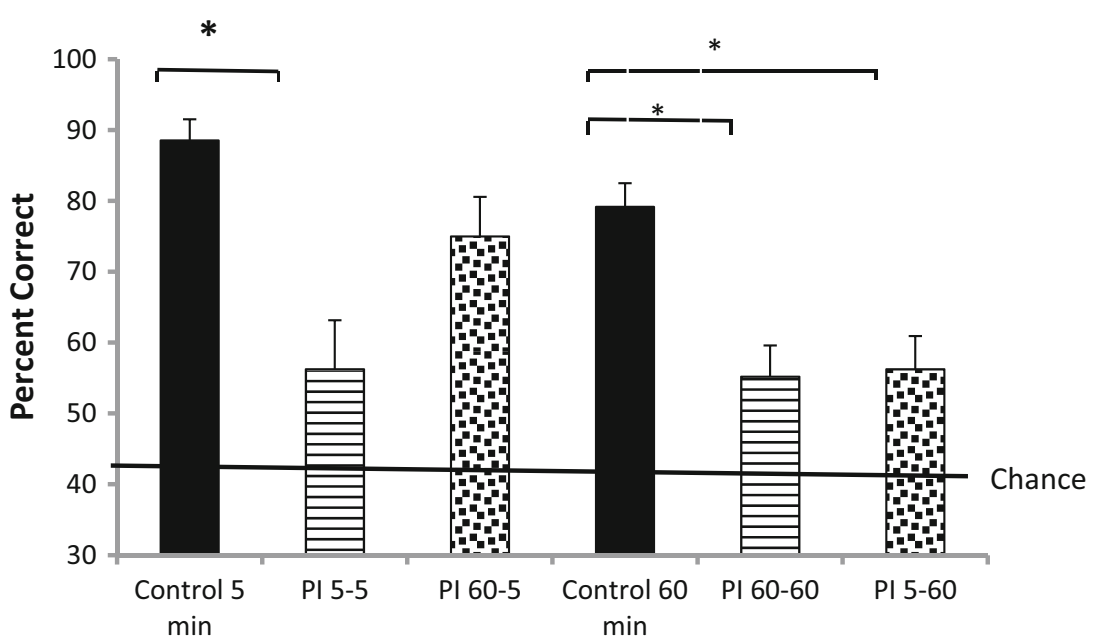

Fig. 2 Percent correct choice is shown for each of the six conditions tested in Experiment 1. Error bars are SEM. $*=p<.05$

comparisons with alpha set at .05 showed a significant difference between Control 5 (88.54\%) and PI 5-5 (56.25\%) but not between Control 5 and PI 60-5 (75\%). Significant differences were found between Control 60 (79.17\%) and PI 60-60 (55.21\%) and between Control 60 and PI 5-60 (56.25\%). Tests of each mean against the chance level of correct performance on the first four choices $(41.38 \%)$ showed that accuracy was significantly better than chance in all six conditions, $t(11) \geq 2.25, p<.05$. The chance level of $41.38 \%$ is based on the assumption that rats rarely make an error by re-entering an arm previously visited. Thus, as rats enter successive arms in the test phase, the probability of choosing a correct arm by chance decreases (Olton \& Samuelson, 1976).

\section{Discussion}

The results of Experiment 1 showed that PI appeared in rat spatial memory using a one-trial procedure in which rats were forced to enter different sets of arms in an interference study phase and in a target study phase before a retention test for the arms entered in the target phase. Under this two-study-phase condition, rats' accuracy in choosing correct arms not visited in the target phase was consistently lower in PI conditions than in target-phase-only control conditions. That rats were choosing the correct arms based on target phase memory even on PI trials was shown by the fact that all of the retention test percent correct scores were significantly better than chance performance. However, the extent of PI varied between PI conditions depending on the relative temporal separation between the interference and target phases and between the target phase and retention test. Rats failed to show a significant difference between the PI $60-5$ and the Control 5 condition, but the other PI conditions, PI 5-5, PI 60-60 and PI 5-60, all showed significant PI effects. Similar findings were reported by Cohen, Sturdy, and Hicks (1996) of little PI when a long interval of 30 min was inserted between study phases and a short interval of 2 min was inserted between the target study phase and the retention test. When these intervals were equal or the inter-study-phases interval was shorter than the retention interval, substantial PI was found.

These findings agree well with the temporal discrimination hypothesis (D'Amato, 1973; Worsham, 1975). It predicted that the lowest amount of PI would be seen in the condition that maximized the ratio between the time interval separating the interference phase and the retention test and the time interval separating the target phase and the retention test. That ratio was 13 in the PI $60-5$ condition but 2 or less in the other three PI conditions, PI 5-5, PI 60-60, and PI 5-60. In the PI 60-5 condition, the remembered time of the interfering phase was far more distant than the remembered time of the target phase. In the other three PI conditions, the relative remembered times of the interfering and target memories were much closer together.

In summary, clear evidence of PI was shown using a onetrial multi-phase procedure. Using this paradigm, one factor was shown to yield protection from or release from PI. Separating the time of the interfering phase from the time of the target phase relative to the retention interval statistically eliminated PI. Having clearly shown robust PI in the PI 5-5 condition, this procedure was used in subsequent experiments to find out if variables other than temporal separation can produce release from PI.

\section{Experiment 2}

This experiment examined the effects of contextual change on $\mathrm{PI}$ in rat spatial memory. Context is defined as the immediate environment in which testing takes place. Reinstatement of the context in which a memory was encoded has been shown to improve human retention (Murnane, Phelps, \& Malmberg, 
1999; Smith \& Vela, 2001). Pavlovian experiments have shown that rats conditioned in context $\mathrm{A}$ and extinguished in context $\mathrm{B}$ will make the conditioned response when reintroduced to context A (the renewal effect; Bouton 1993, 2004). Cheng (2005) showed that training bees in different visual contexts eliminated retroactive interference between opposing spatial memories. Recent experiments with pigeons (Roberts, Macpherson, \& Strang, 2016) and rats (Roberts, Guitar, Marsh, \& MacDonald, 2016) have shown that contextual change controls access to working and reference memories. Experiments in which rats were trained to make alternating arm choices in a T-maze have found release from PI when the context between an interfering trial and a study trial was changed by turning room lights on and off or by changing from a slow to fast auditory click rate (Grant, 1980). Similarly, Beracochea, Lescaudron, Tako, Verna, and Jaffard (1987) showed that placing a white cardboard rectangle into a T-maze between trials enhanced spontaneous alternation in mice. Cohen et al. (1994) found a transient reduction in PI on the radial maze when arm cues and number of reward pellets varied between trials but not when extramaze landmark cues changed between trials. Given these findings, it was thought that a change in context might produce a release from PI in rats on the radial maze.

Context was manipulated along three different sensory dimensions, brightness, tactile surface, and odor. The surface of the arms and center of the maze could be changed from gray to black in brightness and from smooth to rough in texture. Given rats' excellent memory for odors (April, Bruce, \& Galizio, 2013), different scents were placed in the reward cups to further differentiate contexts. On control trials, rats were tested for retention of spatial memory over $5 \mathrm{~min}$ with the same context present on the study phase and the retention test. There were four types of PI trials. On two PI trials, the same context was present on the interfering phase, the target study phase, and the retention test. On two other PI trials, the context was changed between the interfering phase and the target study phase, with the target study phase context maintained on the retention test. If contextual change produces a release from PI, we would expect to find better retention in the context change PI conditions, relative to the control conditions, than in the same context PI conditions.

\section{Method}

The same rats used in Experiment 1 were tested in Experiment 2. The maze used was the same as that in Experiment 1, but its brightness and texture could be changed from gray-smooth to black-rough by placing covers on the arms of the maze and the central platform that were made of a black plastic material that had a stippled surface. Two sets of reward cups were used, each with a wire screen insert on which two reward pellets could be placed. Under the wire screen, an odorant was placed, anise in one set of eight cups and sumac in the other set of eight cups. These reward cups could be attached to the ends of the maze arms with Velcro to establish one odor context or the other. A reward cup containing the relevant odor was also placed in the central hub of the maze.

Two different contexts were used on different control trials. On one control trial, the target study phase and retention test context was black-rough-anise (Control BRA $\rightarrow$ BRA) and on the other control trial, the target study phase and retention test context was gray-smooth-sumac (Control GSS $\rightarrow$ GSS). These two contexts were also maintained during the interference, target study, and test phases of two PI conditions (PI BRA $\rightarrow$ $\mathrm{BRA} \rightarrow \mathrm{BRA}$ and PI GSS $\rightarrow$ GSS $\rightarrow$ GSS). On the other two PI conditions, however, the context was switched between the interference phase and the target study phase (PI GSS $\rightarrow$ $\mathrm{BRA} \rightarrow \mathrm{BRA}$ and PI BRA $\rightarrow \mathrm{GSS} \rightarrow \mathrm{GSS}$ ). Rats were tested on each condition over 6 days in the order Control BRA $\rightarrow$ BRA, Control GSS $\rightarrow$ GSS, PI BRA $\rightarrow$ BRA $\rightarrow$ BRA, PI $\mathrm{GSS} \rightarrow \mathrm{GSS} \rightarrow \mathrm{GSS}$, PI BRA $\rightarrow \mathrm{GSS} \rightarrow \mathrm{GSS}$, and PI GSS $\rightarrow$ BRA $\rightarrow$ BRA. Testing then continued for another 6 days, with the order of conditions reversed. The time intervals between the study phases and between the target study phase and the retention test were both $5 \mathrm{~min}$.

\section{Results and discussion}

The percentage of correct choices in the first four arm entries is shown for each condition in Fig. 3. These data clearly suggest that PI was found, as accuracy was lower on all of the PI conditions than on both of the control conditions. However, performance was significantly better than chance in all six conditions, $t(11) \geq 5.12, p<.01$. Further, there appears to be little difference between the PI conditions that changed context and those that did not. A one-way ANOVA showed a significant effect of condition, $F(5,55)=8.45, p<.01, \eta_{\rho}^{2}$ $=.44$. Post-hoc comparisons revealed significant differences between Control BRA $\rightarrow$ BRA and both PI BRA $\rightarrow$ BRA $\rightarrow$ BRA and PI GSS $\rightarrow$ BRA $\rightarrow$ BRA. Similarly, significant differences were found between Control GSS $\rightarrow$ GSS and both PI $\mathrm{GSS} \rightarrow \mathrm{GSS} \rightarrow \mathrm{GSS}$ and $\mathrm{PI} \mathrm{BRA} \rightarrow \mathrm{GSS} \rightarrow \mathrm{GSS}$.

Clearly these results failed to show that context change produced release from PI. Equivalent PI was found with and without a context change between the interference and study phases. It could be argued that these changes in context were not sufficient to cause release from PI. However, the contextual changes would seem substantial as they involved changes in three important sensory dimensions.

\section{Experiment 3}

In Experiment 3, we manipulated another property of the maze between the interference and target phases of PI trials, 


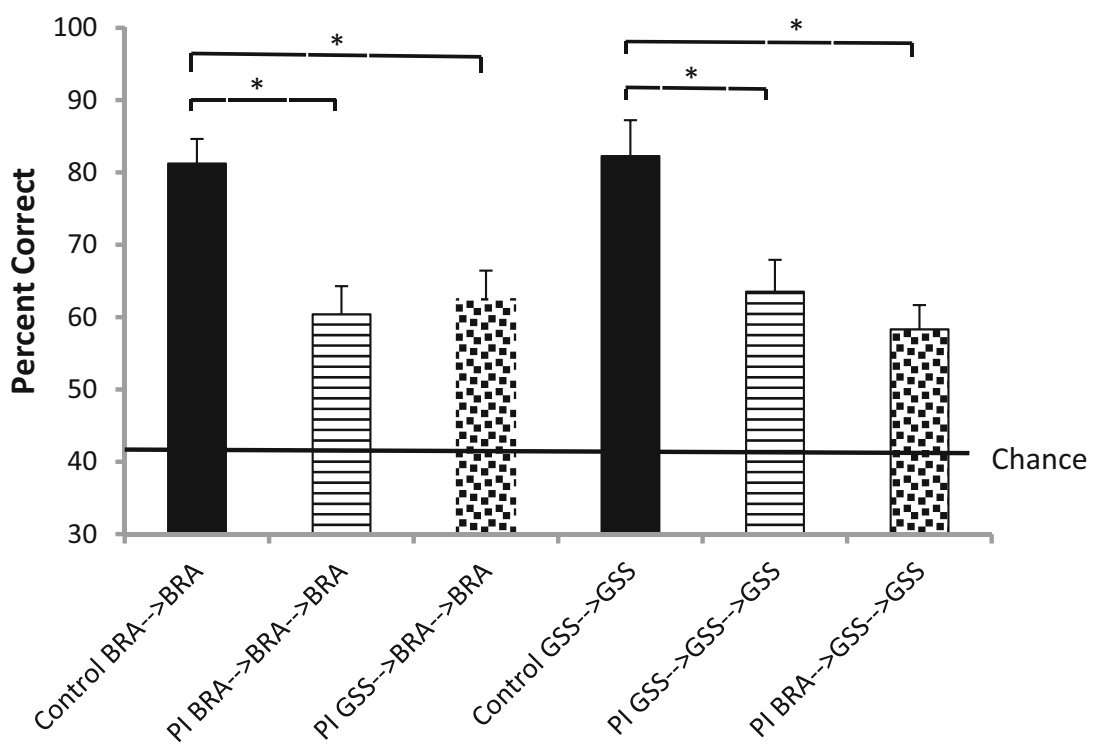

Fig. 3 Percent correct choice is show for each of the six conditions tested in Experiment 2. Error bars are SEM. BRA Black Rough Anise, GSS Gray Smooth Sumac. $*=p<.05$

the pattern of arms visited. In Experiments 1 and 2, the pattern of arms visited in the interference and target study phases was random and varied between rats and trials. In addition to a PI Random condition like those used in the first two experiments, this experiment also examined performance in PI and control Alternate and PI and control Adjacent conditions. In the PI Alternate condition, rats were forced to visit arms for reward that alternated with closed arms in the interference phase and the opposite alternating pattern in the study phase; thus, the distance between one baited arm and next closest baited arm was always $90^{\circ}$. In the PI Adjacent condition, rats were forced to visit a block of four adjacent arms on one side (no closed arms separating baited arms) of the maze in the interference phase and the block of four adjacent arms on the opposite side of the maze in the study phase.

We reasoned that the use of a recognizable pattern of visited arms might facilitate discrimination between interference and study memories and thus produce release from PI. It was thought that use of the adjacent pattern in particular might produce release from PI. If rats in this condition could associate interfering arms with cues on one side of the maze and testing room and target study arms with a different set of cues on the other side of the maze and testing room, confusion between interference and study arms visited would be far less than when random patterns were used.

\section{Method}

The same rats and maze used in the first two experiments were used in Experiment 3. There were six conditions tested, Control Random, PI Random, Control Alternate, PI Alternate, Control Adjacent, and PI Adjacent. Rats were tested daily on one condition in this order and then tested again on each condition in the reverse order. As in the preceding experiment, PI trials involved an interference phase, a study phase, and a retention test (see Fig. 4), and control trials involved only the study phase and the retention test. The interval between phases was always 5 min. On sessions using random patterns, the pattern of forced arm choices was chosen by a random number generator for each rat as in Experiments 1 and 2. There were only two possible patterns of alternating arms. Rats were tested with one pattern as the interfering pattern and the other as the study pattern in the first block of six trials, and this order of interfering and study patterns was reversed in the second block of six trials. There are four different possible adjacent arm patterns. Rats were tested using two of these patterns, with one used in the interference phase and the other used in the study phase in the first block of six trials. The use of these patterns was reversed between the interference phase and the study phase in the second block of trials.

\section{Results}

Performance in Experiment 3 is shown in Fig. 5. Perhaps surprisingly, accuracy varied across arm patterns, but PI was observed with each pattern. In particular, when rats were tested in the Control Alternate condition, only one arm was incorrectly entered in 24 retention tests. A one-way ANOVA showed that performance differed significantly across the six conditions, $F(5,55)=15.53, p<.01, \eta_{\rho}{ }^{2}=.58$. Post-hoc tests showed significant differences between the Control Random (89.58\%) and PI Random (70.83\%) conditions, between the Control Adjacent (75\%) and PI Adjacent (58.33\%) 
PI Random

Interference Phase

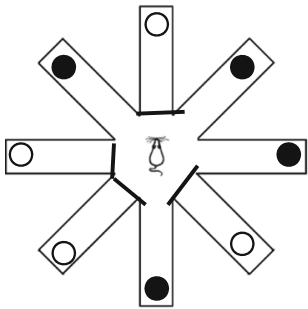

Target Study Phase

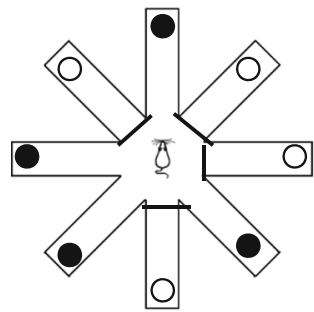

Retention Test Phase

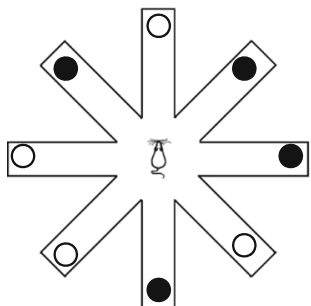

PI Alternate

Interference Phase

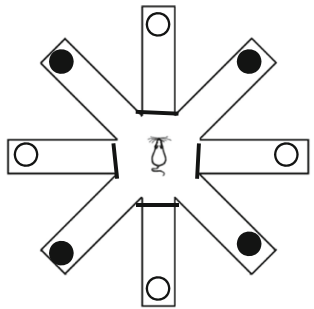

Target Study Phase

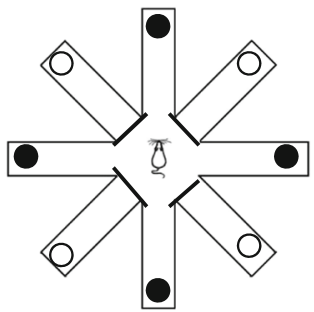

Retention Test Phase

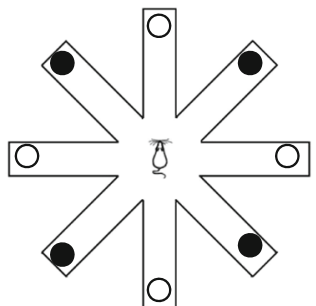

PI Adjacent

Interference Phase

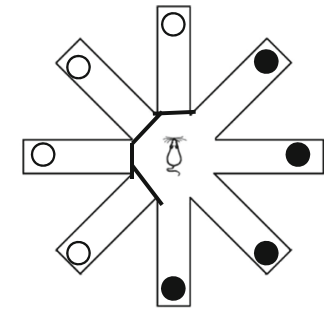

Target Study Phase

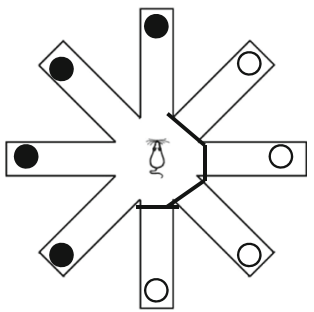

Retention Test Phase

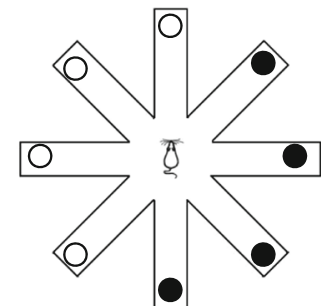

Fig. 4 Diagram showing the baited and empty arms during the three phases of Experiment 3 for the Random Arms, Alternate Arms, and Adjacent Arms conditions. Lines across arm entrances show blocked arms. $\bullet=$ baited arm; $\circ=$ empty arm

conditions, and between the Control Alternate $(98.96 \%)$ and PI Alternate $(72.92 \%)$ conditions. Once again, performance under all six conditions was significantly better than chance, $t(11) \geq 6.04, p<.05$.

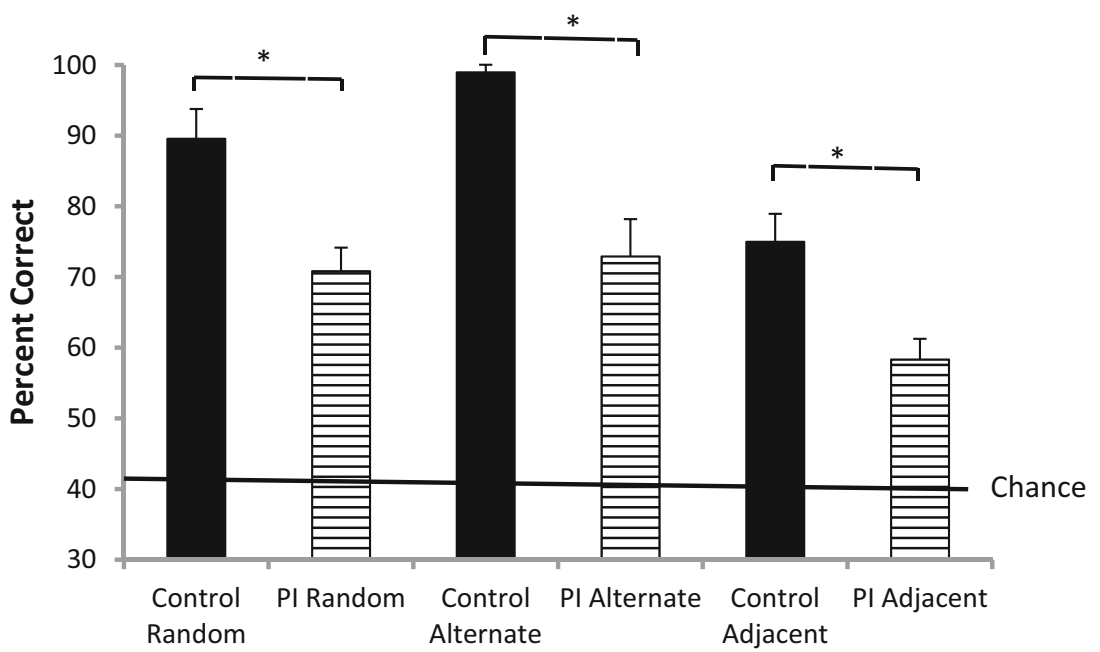

Fig. 5 Percent correct choice is shown for each of the six conditions tested in Experiment 3. Error bars are SEM. $*=p<.05$ 


\section{Discussion}

These results show that accuracy on radial maze retention tests varies considerably with the pattern of forced arm entries on the study phase. In particular, accuracy was substantially higher on the Control Alternate condition (98.96\%) than on the Control Adjacent condition (75\%). A strong preference for $90^{\circ}$ turns after exiting one arm may be partially responsible for this difference. However, rats had to know which of the arms was correct on the first choice before executing a series of $90^{\circ}$ turns. Similar results were reported by Hoffman and Maki (1986), who found better memory for a cross (alternating) pattern than for a side (adjacent) pattern on a radial maze. With regard to the main purpose of this experiment, it is interesting that PI was found with all three maze patterns. Although accuracy may be strongly influenced by the pattern of arms tested, it appears that PI was common to testing with all three patterns. Thus, no evidence of release from PI was found with any of the patterns.

\section{Experiment 4}

It is well known that rats learn about the reward contents of the arms on a radial maze and use this information to organize their arm visits. Rats that repeatedly visit arms on the radial maze with different amounts of food in fixed locations develop a pattern of visiting the arms in descending quantity of food (Hulse \& O'Leary, 1982; Roberts, 1992), and rats learn the locations of different flavored rewards on the radial maze (Babb \& Crystal, 2006). Reward pellets purchased from a commercial supplier were used in Experiments 1-3. In Experiment 4, we asked whether the use of more palatable food rewards would influence PI effects on the radial maze. The rewards used were pieces of cheese and chocolate. Macuda and Roberts (1995) showed that rats have a clear preference for cheese over chocolate and for chocolate over standard reward pellets. Control conditions were tested with each reward. Four types of PI trials were tested. On two types of PI trials, the same reward was used in the interference phase, the target phase, and the retention test. On two other types of PI trials, different rewards were given on forced visits to arms in the interference and target study phases, either cheese $\rightarrow$ chocolate or chocolate $\rightarrow$ cheese, with the study target reward placed in the correct arms on the retention test.

This experiment then examined whether release from PI might occur when more palatable rewards were found on the maze arms, both when the type of reward was the same on the interference and target study phases and when the type of reward differed between phases. It is possible that memories from the interference and target phases may be more discriminable with more palatable rewards that differ between phases.

\section{Method}

The same rats and maze used in the preceding experiments were used in Experiment 4. The rats were tested on single daily trials using the same procedure as used in Experiments 1-3 but with different rewards placed in the food cups at the end of the maze arms. A different random selection of arms for forced choices was used on each trial for each rat. On PI trials, either the same reward was used in the interference phase, the study phase, and the retention test or the reward changed between the interference phase and the study phase. On the first 6 days of testing, the order of conditions tested was Control Cheese, Control Chocolate, PI Cheese $\rightarrow$ Cheese $\rightarrow$ Cheese, PI Chocolate $\rightarrow$ Chocolate $\rightarrow$ Chocolate, PI Cheese $\rightarrow$ Chocolate $\rightarrow$ Chocolate, and PI Chocolate $\rightarrow$ Cheese $\rightarrow$ Cheese. On the subsequent six trials of testing, this order was reversed. The interval between phases was always $5 \mathrm{~min}$.

The cheese reward used was two small pieces $(.05 \mathrm{~g})$ of medium cheddar cheese placed in each of the appropriate food cups. The chocolate reward was two Hershey's milk chocolate Chipits placed in each of the appropriate food cups. The cup in the center of maze also contained the same reward as found on the arms.

\section{Results}

The percentage of correct choices made on each condition is shown in Fig. 6. When cheese was the reward on the retention test, accuracy in the PI Cheese $\rightarrow$ Cheese $\rightarrow$ Cheese condition $(64.58 \%)$ was lower than in the Control Cheese condition $(71.88 \%)$ but not in the PI Chocolate $\rightarrow$ Cheese $\rightarrow$ Cheese condition (72.92\%). When chocolate was the reward on the retention test, rats showed a drop in performance on the $\mathrm{PI} \rightarrow$ Chocolate $\rightarrow$ Chocolate $\rightarrow$ Chocolate condition (69.79\%) relative to the Control Chocolate condition (79.17\%). There was an even more substantial difference between the PI Cheese $\rightarrow$ Chocolate $\rightarrow$ Chocolate $(53.12 \%)$ condition and the Control Chocolate condition. A one-way ANOVA comparing all six conditions yielded a significant effect, $F(5,55)=8.22, p<.01$, $\eta_{\rho}{ }^{2}=.43$. Post-hoc tests showed a significant difference only between the Control Chocolate and PI Cheese $\rightarrow$ Chocolate $\rightarrow$ Chocolate conditions. As in the preceding experiments, performance on the retention test significantly exceeded chance in all conditions, $t(11) \geq 2.68, p<.05$.

\section{Discussion}

These findings suggest that the use of more palatable rewards (cheese and chocolate) reduced and statistically eliminated PI effects. That is, neither the PI Cheese $\rightarrow$ Cheese $\rightarrow$ Cheese condition nor the PI Chocolate $\rightarrow$ Chocolate $\rightarrow$ Chocolate condition differed significantly from the relevant control condition. Thus, the use of more palatable rewards may enhance the 


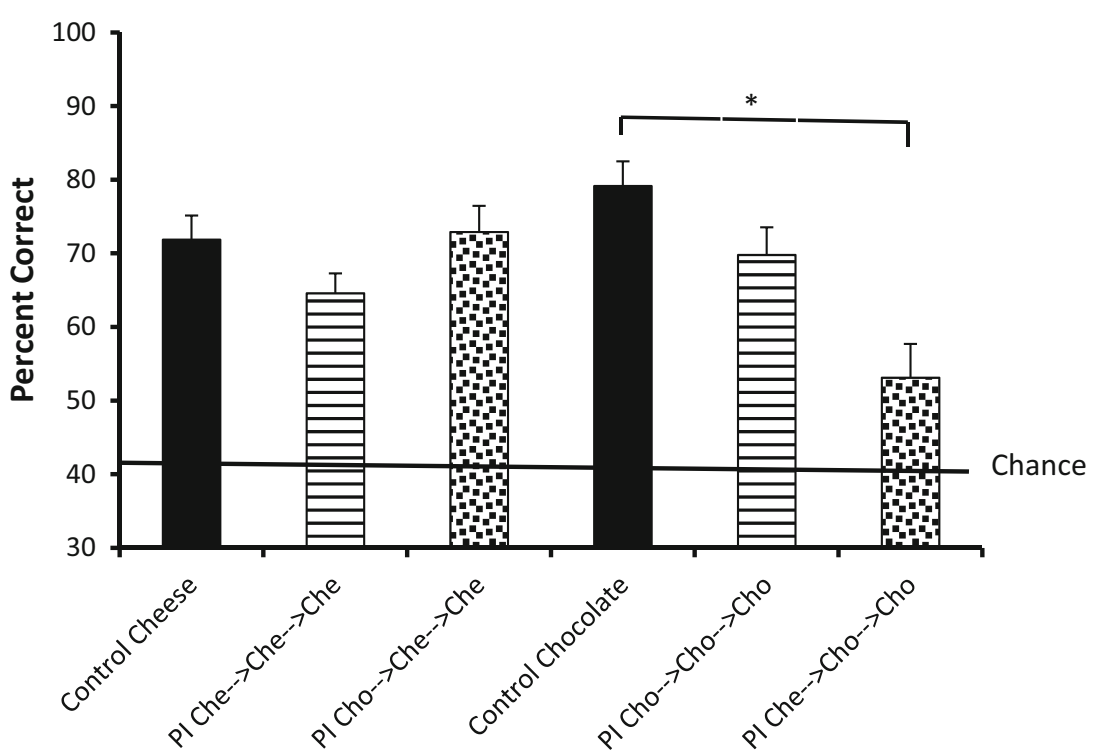

Fig. 6 Percent correct choice is shown for each of the six conditions tested in Experiment 4. Error bars are SEM. Che cheese, Cho chocolate. * $=$ p $<.05$

discrimination of study phase memory from interference phase memory. The finding that accuracy in the PI Chocolate $\rightarrow$ Cheese $\rightarrow$ Cheese condition was equivalent to that in the Control Cheese condition might be explained by memories for cheese arms being more salient than memory for chocolate arms. The same argument can be used to account for the PI effect seen in the PI Cheese $\rightarrow$ Chocolate $\rightarrow$ Chocolate condition. If rats' memory for the initially visited cheese arms was particularly strong, this would lead them to avoid those arms and incorrectly revisit the chocolate arms they were forced to enter in the target study phase. Unfortunately, a major problem with this argument is the observation that rats did not show higher retention on Control Cheese trials (71.88\%) than on Control Chocolate trials $(79.17 \%)$. Further, recent findings reported by Smith, Dalecki, and Crystal (2017) failed to show a difference in memory over $24 \mathrm{~h}$ between rats rewarded on maze arms with chow and chocolate, suggesting that reward value does not affect strength of memory encoding. Nonetheless, these results suggest that under certain conditions, the manipulation of salient rewards does lead to release from PI in rat spatial memory.

\section{General discussion}

These experiments demonstrated PI within a single trial of spatial memory testing on the radial maze. Rats showed substantial forgetting of a set of four target arms visited when they were previously forced to visit an alternate set of four interfering arms. Control tests run without visits to interfering arms yielded higher levels of retention. However, in all the experiments, rats showed significantly higher levels of accuracy on PI trials than expected by chance selection of arms. Thus, the interference phase did not totally eliminate retention of the target arms.

The primary goal of this series of experiments was to find out if certain testing conditions would produce release from PI. In human working memory experiments, changes in a variety of dimensions cause release from PI (see Introduction). Thus, several variables were manipulated between the interference study phase and the target study phase to see whether some changes would protect memory of the target phase arm entries from PI. It was found that some variables yielded release from PI but others did not. An examination of this pattern of findings may give us some clues about how rats encode arm location memories on the radial maze.

Experiment 1 clearly showed the importance of the time interval between the interference phase and the retention test relative to the time interval between the target phase and the retention test. Proactive interference was statistically eliminated in the PI 60-5 condition when 60 min separated the interfering phase and the target phase and the retention interval was $5 \mathrm{~min}$. It is interesting to note that neither of these time intervals alone caused release from PI. That is, significant PI was found when the separation of the interference and target phases was 60 min in the PI 60-60 condition and when the retention interval was 5 min in the PI 5-5 condition. The best account of this pattern is offered by the temporal discrimination hypothesis (D'Amato, 1973; Worsham, 1975), which holds that confusion between an initial interfering memory and a subsequent target memory should be reduced when the interference memory is remembered as far more distant in time from the retention test than the target memory. Thus, memory of arm entries that occurred 65 min ago should be easily discriminated from memory of arm entries that occurred only 5 min ago.

Relative temporal separation appears to be one factor that produces release from PI in rat spatial memory. Because clear 
evidence of PI was found in the PI 5-5 condition, we can ask further if change in variables within the maze itself or in the pattern of arm selection might produce release from PI, even when the time intervals between phases are short. Thus, Experiments 2-4 all used the PI 5-5 paradigm and a 5-min retention interval on control trials. In Experiment 2, a substantial change in the within-maze context was introduced between the interference phase and the target phase and retention test. The context was changed along three different sensory dimensions, brightness of the floor (gray vs. black), tactile sensation of the floor (smooth vs. rough) and odor of the food cups (anise vs. sumac). This change in context produced no release from PI; equivalent PI was found when the context was and was not changed, relative to control performance. This failure to find a release from PI is surprising given that similar changes in context have shown a significant reduction in PI in single alternation tasks with rats (Grant, 1980) and mice (Beracochea et al., 1987). However, single alternation in a T-maze involves memories for locations that are $180^{\circ}$ apart. On the radial maze, arms are much closer to one another in space. An interference arm entry and a target arm entry may be adjacent (only $45^{\circ}$ apart). It could be that context change promotes better discrimination between memories of widely separated spatial locations than between memories of closely adjacent multiple spatial locations.

The question of spatial separation and maze pattern was addressed in Experiment 3. It was asked whether rats might discriminate better between interference and target memories if these memories followed a predictable pattern or were spatially separated. In a further search for conditions that might produce release from PI, rats were given interference trials on which they were forced to enter alternating arms separated by $90^{\circ}$ or to enter four adjacent arms on one side of the maze. The target phase then involved forced entries into the remaining alternating arms or the remaining adjacent arms on the other side of the maze. It was hypothesized that the adjacent arms condition in particular might produce a release from PI because rats could associate arm entrances in the interference and target phases with extramaze cues on different sides of the testing room. The results clearly did not correspond to this hypothesis, as the rats showed significant PI in both the alternate-arm condition and the adjacent-arm condition that was equivalent to the PI found in a random-arm condition. Further, in contrast with the idea that rats would be able to better remember arm entrances adjacent to one another, Control Adjacent and PI Adjacent conditions showed the lowest accuracy. By contrast, rats were nearly errorless in the Control Alternate condition. Although failing to show release from PI, these findings suggest that preferred patterns of turning between exiting one arm and entering another may play an important role in spatial memory and should be further investigated.
In Experiment 4, the arms of the radial maze were baited with cheese and chocolate to examine PI effects when more palatable rewards were used. The interesting finding here was that no evidence of PI was found when either cheese or chocolate was the reward in both the interference phase and the target phase. This was also the case in the PI Chocolate $\rightarrow$ Cheese $\rightarrow$ Cheese condition. On the other hand, significant PI was found in the PI Cheese $\rightarrow$ Chocolate $\rightarrow$ Chocolate condition relative to the Control Chocolate condition.

This pattern of findings is of considerable importance because it suggests that the nature of the reward found on the end of maze arms may be a key factor in spatial memory encoding. In reward preference tests, Macuda and Roberts (1995) found that rats showed a strong preference for cheese over chocolate and for chocolate over reward pellets. Further, when these different rewards were placed on different arms of a 12-arm radial maze, rats chunked their arm visits by going to the cheese arms first, the chocolate arms second, and the pellet arms last. Thus, rats may encode arm locations visited by the type of reward found on an arm when the rewards are highly salient. The failure to find PI in Experiment 4 when either cheese or chocolate was used in all phases of a PI trial can be explained by place-reward encoding that allowed rats to distinguish between target and interference memories. The fact that PI was commonly seen in the PI 5-5 conditions tested in Experiments 1-3 may have been the result of relatively weaker place-reward encoding caused by the use of lowpreference reward pellets. Finally, rats strong preference for cheese over chocolate may explain the PI effect seen in the PI Cheese $\rightarrow$ Chocolate $\rightarrow$ Chocolate condition in Experiment 4 . If arm locations where cheese was found in the interference phase were encoded better than arm locations where chocolate was found in the target phase, rats better memory for cheese locations would cause them to revisit target chocolate locations on the retention test. By the same reasoning, the absence of PI found in the PI Chocolate $\rightarrow$ Cheese $\rightarrow$ Cheese condition is expected. However, these arguments are complicated by the fact that that the Control Cheese condition performed less accurately than the Control Chocolate condition in Experiment 4 and by recent evidence that quality of reward may not affect retention on the radial maze (Smith et al., 2017).

The findings of these experiments shed some light on factors important for the encoding of arm visits on the radial maze and perhaps on location memories in more natural settings. Failure to find PI in some experiments suggested that certain factors play no role in place memory encoding. Variation in maze context along several sensory dimensions caused no release from PI. Forcing rats to enter arms that formed a predictable pattern or that could be easily associated with different extramaze cues also failed to cause release from PI. By contrast, manipulation of time and reward led to conditions that produced release from PI. Release from PI was found 
when memory of target arm entries was recent and memory of interfering arm entries was distant in time (Condition PI 605). The importance of location-reward encoding was shown by the finding that PI did not occur when preferred highly palatable rewards were used (cheese and chocolate) but did occur when the more preferred reward (cheese) was placed on arms in the interference phase and the less preferred reward (chocolate) was placed on the arms in the target phase. Release from PI then is found in rats as well as people and the release-from-PI paradigm may be an important procedure for discovering how memories are encoded by rats and other species.

Acknowledgements Support for this research was provided by a Discovery Grant from the Natural Sciences and Engineering Research Council of Canada. The animals used in these experiments were maintained and tested in compliance with the regulations of the Western University Animal Care Committee and the Canadian Council on Animal Care.

\section{References}

April, L. B., Bruce, K., \& Galizio, M. (2013). The magic number 70 (plus or minus 20): Variables determining performance in the rodent odor span task. Learning and Motivation, 44, 143-158.

Babb, S. J., \& Crystal, J. D. (2006). Episodic-like memory in the rat. Current Biology, 16, 1317-1321.

Beracochea, D., Lescaudron, L., Tako, A., Verna, A., \& Jaffard, R. (1987). Build-up and Release from proactive interference during chronic ethanol consumption in mice: A behavioral and neuroanatomical study. Behavioral Brain Research, 25, 63-74.

Bouton, M. E. (1993). Context, time, and memory retrieval in the interference paradigms of Pavlovian learning. Psychological Bulletin, $114,80-99$

Bouton, M. E. (2004). Context and behavioral processes in extinction. Learning and Memory, 11, 486-494.

Burr, D. E. S., \& Thomas, D. R. (1972). Effect of proactive inhibition upon the post-discrimination generalization gradient. Journal of Comparative and Physiological Psychology, 81, 444-448.

Cheng, K. (2005). Context cues eliminate retroactive interference effects in honeybees (Apis mellifera). Journal of Experimental Biology, 208, 1019-1024.

Cohen, J. S., Reid, S., \& Chew, K. (1994). Effects of varying trial distribution, intra- and extramaze cues, and amount of reward on proactive interference in the radial maze. Animal Learning \& Behavior, 22, 134-142.

Cohen, J. S., Sturdy, C., \& Hicks, M. (1996). Intratrial proactive interference in rats' serial alternation performance in the radial maze. Animal Learning \& Behavior, 24, 300-309.

D'Amato, M. R. (1973). Delayed matching and short-term memory in monkeys. In G. H. Bower (Ed.), The psychology of learning and motivation: Advances in research and theory (Vol. 7, pp. 227-269). Academic Press: New York, NY.

Darling, S., \& Valentine, T. (2005). The categorical structure of semantic memory for famous people: A new approach using release from proactive interference. Cognition, 96, 35-65.

Devkar, D. T., \& Wright, A. A. (2016). Event-based proactive interference in rhesus monkeys. Psychonomic Bulletin Review, 23, 1474 1482.
Dillon, R. F., MCormack, P. D., Petrusic, W. M., Cook, G. M., \& LaFleur, L. (1973). Release from proactive interference in compound and coordinate bilinguals. Bulletin of the Psychonomic Society, 2, 293-294.

Dunnett, S. B., \& Martel, F. L. (1990). Proactive interference effects on short-term memory in rats: I. Basic parameters and drug effects. Behavioral Neuroscience, 104, 655-665.

Gleitman, H., \& Jung, L. (1963). Retention in rats: The effect of proactive interference. Science, 142, 1683-1684.

Goggin, J., \& Wickens, D. D. (1971). Proactive interference and language change in short-term memory. Journal of Verbal Learning and Verbal Behavior, 10, 453-458.

Grant, D. S. (1975). Proactive interference in pigeon short-term memory. Journal of Experimental Psychology: Animal Behavior Processes, 104, 207-220.

Grant, D. S. (1980). Delayed alternation in the rat: Effect of contextual stimuli on proactive interference. Learning and Motivation, 11, 339-354.

Grant, D. S. (1981). Intertrial interference in rat short-term memory. Journal of Experimental Psychology: Animal Behavior Processes, 7, 217-227.

Hoffman, N., \& Maki, W. S. (1986). Two sources of proactive interference in spatial working memory: Multiple effects of repeated trials on radial maze performance by rats. Animal Learning \& Behavior, $14,65-72$.

Hulse, S. H., \& O’Leary, D. K. (1982). Serial pattern learning: Teaching an alphabet to rats. Journal of Experimental Psychology: Animal Behavior Processes, 8, 260-273.

Jitsumori, M., Wright, A. A., \& Shyan, M. R. (1989). Buildup and release from proactive interference in a rhesus monkey. Journal of Experimental Psychology: Animal Behavior Processes, 15, 329-337.

Keppel, G., \& Underwood, B. J. (1962). Proactive inhibition in shortterm retention of single items. Journal of Verbal Learning and Verbal Behavior, 1, 153-161.

Kraemer, P. J. (1984). Forgetting of visual discriminations by pigeons. Journal of Experimental Psychology: Animal Behavior Processes, 10, 530-542.

Kroll, N. E., Bee, J., \& Gurski, G. (1973). Release of proactive interference as a result of changing presentation modality. Journal of Experimental Psychology, 98, 131-137.

Macuda, T., \& Roberts, W. A. (1995). Further evidence for hierarchical chunking in rat spatial memory. Journal of Experimental Psychology: Animal Behavior Processes, 21, 20-32.

Murnane, K., Phelps, M. P., \& Malmberg, K. (1999). Context-dependent recognition memory: The ICE theory. Journal of Experimental Psychology: General, 128, 403-415.

Olton, D. S. (1977). Spatial memory. Scientific American, 236, 82-98.

Olton, D. S. (1978). Characteristics of spatial memory. In S. H. Hulse, H. Fowler, \& W. K. Honig (Eds.), Cognitive processes in animal behavior (pp. 341-373). Erlbaum: Hillsdale, NJ.

Olton, D. S., \& Samuelson, R. J. (1976). Remembrance of places passed: Spatial memory in rats. Journal of Experimental Psychology: Animal Behavior Processes, 2, 97-116.

Postman, L. (1962). The temporal course of proactive inhibition for serial lists. Journal of Experimental Psychology, 63, 361-369.

Roberts, W. A. (1980). Distribution of trials and intertrial retention in delayed matching to sample with pigeons. Journal of Experimental Psychology: Animal Behavior Processes, 6, 217-237.

Roberts, W. A. (1992). Foraging by rats on a radial maze: Learning, memory, and decision rules. In I. Gormezano \& E. A. Wasserman (Eds.), Learning and memory: The Behavioral and biological substrates (pp. 7-23). Erlbaum: Hillsdale, NJ.

Roberts, W. A., \& Dale, R. H. I. (1981). Remembrance of places lasts: Proactive inhibition and patterns of choice in rat spatial memory. Learning and Motivation, 12, 261-281.

Roberts, W. A., Guitar, N. A., Marsh, H. L., \& MacDonald, H. (2016). Memory systems in the rat: Effects of reward probability, context, 
and congruency between working and reference memory. Animal Cognition, 19, 593-604.

Roberts, W. A., \& Kraemer, P. J. (1982). Some observations of the effects of intertrial interval and delay on delayed matching to sample in pigeons. Journal of Experimental Psychology: Animal Behavior Processes, 8, 342-353.

Roberts, W. A., Macpherson, K., \& Strang, C. (2016). Context controls access to working and reference memory in the pigeon (Columba livia). Journal of the Experimental Analysis of Behavior, 105, 184-193.

Smith, A. E., Dalecki, S. J., \& Crystal, J. D. (2017). A test of the rewardvalue hypothesis. Animal Cognition, 20, 215-220.

Smith, S. M., \& Vela, E. (2001). Environmental context-dependent memory: A review and meta-analysis. Psychonomic Bulletin \& Review, $8,203-220$.
Turvey, M. T., \& Egan, J. (1969). Contextual change and release from proactive interference in short-term verbal memory. Journal of Experimental Psychology, 81, 396-397.

Wickens, D. D. (1970). Encoding categories of words: An empirical approach to meaning. Psychological Review, 77, 1-15.

Wickens, D. D., Clark, S., Hill, S. E., \& Wittlinger, R. P. (1968). Grammatical class as an encoding category in short-term memory. Journal of Experimental Psychology, 78, 599-604.

Worsham, R. W. (1975). Temporal discrimination factors in the delayed matching-to-sample task in monkeys. Animal Learning \& Behavior, 3, 93-97.

Wright, A. A., Katz, J. S., \& Ma, W. J. (2012). How to be proactive about interference: Lessons from animal memory. Psychological Science, 23, 453-458. 